\title{
Caracterización del crecimiento de bovinos cebú en pruebas de comportamiento en pastoreo
}

\section{Characterization of growth of Cebu cattle in testing performance on grazing}

\author{
Joel Domínguez-Viveros $^{1 *}$, Alfredo Ramón Urbina-Valenzuela ${ }^{2}$, Alejandro Palacios-Espinoza ${ }^{3}$, Nicolás \\ Callejas-Juárez ${ }^{1}$, Juan Ángel Ortega-Gutiérrez ${ }^{1}$, José Luis Espinoza-Villavicencio ${ }^{3}$, Yamariz \\ Padrón-Quintero ${ }^{4}$, Manuel Rodríguez-Castro ${ }^{4}$ \\ ${ }^{1}$ Facultad de Zootecnia y Ecología; Universidad Autónoma de Chihuahua. Periférico Francisco R. Almada Km 1. Chihuahua, \\ Chihuahua, México. CP. 31453. \\ ${ }^{2}$ Facultad de Ciencias Químicas, Universidad Autónoma de Chihuahua. Circuito Universitario 8, Campus Uach II, CP. 31125. \\ Chihuahua, Chihuahua, Mépexico. \\ ${ }^{3}$ Departamento de Zootecnia, Universidad Autónoma de Baja California Sur. Carretera al Sur KM 5.5, Apartado Postal 19-B, \\ CP. 23080. La Paz, Baja California Sur, México \\ ${ }^{4}$ Centro de Investigaciones para el Mejoramiento Animal de la Ganadería Tropical. Ave 101 No. 6214 e/ 62 y 100 Loma de \\ Tierra Cotorro, La Habana, Cuba. \\ *Autor de correspondencia: jodominguez@uach.mx
}

Artículo científico recibido: 03 de julio de 2016, aceptado: 10 de noviembre de 2016

RESUMEN. El objetivo fue caracterizar el crecimiento de bovinos Cebú en pruebas de comportamiento en pastoreo. Se utilizaron 15990 datos de peso vivo, tomados entre 150 y $630 \mathrm{~d}$ de edad, de 3198 machos con cinco pesadas cada uno: 1) peso al destete (PD), 2) peso inicio de prueba (PI), 3) primera pesada (P1), 4) segunda pesada (P2) y 5) peso final de prueba (PF). Se contrastaron los modelos no lineales (MNL): Brody (BRO), Logístico (LOG), von Bertalanffy (BER) y Gompertz (GOM); en dos tipos de análisis (ANA1, ANA2) con NLMIXED de SAS. ANA1, con MNL mixtos (MNLM), al incluir el efecto aleatorio de un coeficiente de regresión; y ANA2 con MNL, sin efectos aleatorios asociados a coeficientes de regresión. La selección del modelo se realizó con los criterios error de predicción promedio; varianza del error de predicción; estadístico Durbin Watson; coeficiente de determinación; criterio de información Akaike y criterio Bayesiano. Los pesos promedio ( $\mathrm{kg}$ ) ajustados al PD, PI, P1, P2 y PF fueron $175.9 \pm 33.4,182.5 \pm 32.1,225.8 \pm$ 49.6, $266.8 \pm 61.9$ y $340.3 \pm 68.1$, respectivamente. Los análisis con MNLM (ANA1) presentaron mejores resultados, siendo el LOG el que presentó mejor ajuste, seguido de GOM, BER y BRO. Los resultados del ANA2 fueron sobre estimados y fuera de contexto para asociarse al crecimiento de bovinos; las curvas de crecimiento generadas con MNLM presentaron buena capacidad predictiva y estuvieron en función del proceso generador de los datos, que se refiere al crecimiento del ganado Cebú.

Palabras clave: modelos no lineales mixtos, ganadería tropical, curvas crecimiento, peso adulto, tasa crecimiento

ABSTRACT. The objective was to characterize the growth of Cebu cattle in grazing performance tests. We used 15990 live weight data, taken between 150 and $630 \mathrm{~d}$ of age, of 3198 males with five weights each: 1) weaning weight (WW), 2) initial test weight (IW), 3) first weighing (W1), 4) second weighing (W2) and 5) final test weight (FW). Nonlinear models (NLM) were compared: Brody (BRO), Logistic (LOG), von Bertalanffy (BER) and Gompertz (GOM). Two types of analyses (ANA1, ANA2) were carried out with the SAS NLMIXED procedure: ANA1 with mixed NLM (MNLM), by including the random effect of a regression coefficient; and ANA2 with MNL, with no random effects associated with regression coefficients. The model was selected using the criteria of mean prediction error; variance of the prediction error; Durbin Watson statistic; coefficient of determination; Akaike information criterion and Bayesian criterion. The mean weights (kg) for WW, IW, W1, W2 and FW were 175.9 \pm 33.4, $182.5 \pm 32.1,225.8 \pm 49.6,266.8$ \pm 61.9 and $340.3 \pm 68.1$, respectively. Analyses with MNLM (ANA1) presented better results, with LOG being the best fit, followed by GOM, BER and BRO. The ANA2 results were overestimated and out of context to be associated 
with bovine growth; the growth curves generated with MNLM showed good predictive capacity and were based on the data generating process, which refers to the growth of Cebu cattle.

Key words: mixed nonlinear models, tropical livestock, growth curves, adult weight, growth rate

\section{INTRODUCCIÓN}

La curva de crecimiento animal se ha conformado y caracterizado por el peso vivo medido a diferentes edades, considerados como datos longitudinales o medidas repetidas de la misma característica, en el mismo individuo. El análisis de datos longitudinales, para generar la curva de crecimiento, se realiza por medio de regresión con modelos lineales y no lineales (Lawrence y Fowler 2002, Thornley y France, 2007). En los modelos no lineales (MNL) los coeficientes de regresión $(\beta i)$, también denominados parámetros de crecimiento, aparecen en forma no lineal en la ecuación, tienen la definición y propiedades de efectos fijos, y poseen una interpretación biológica que facilita el entendimiento del fenómeno (Owens et al. 1993, Motulsky y Christopoulos 2003).

Los MNL han sido evaluados con base en la bondad de ajuste de los datos, la interpretación biológica de los parámetros, y los requerimientos computacionales (Agudelo et al. 2008, Torres et al. 2012); la selección de modelos se realiza con base en la adecuación, que evalúa y jerarquiza el nivel de ajuste de los modelos a los datos analizados; y, la validación, que identifica al modelo que mejor se aproxima al verdadero proceso generador de los datos (Burnham y Anderson 1998). Un mejor ajuste del modelo a los datos, no implica una mejor capacidad predictiva (Zucchini 2000). En la definición, ajuste y validación de los MNL, existen algunas fuentes de variación que no han sido incorporadas, como en el análisis de medidas repetidas se genera una estructura de (co)varianzas que puede inferir en el ajuste y validación de los MNL. Los $\beta$ i asumen que la curva de crecimiento es estándar o única a través de la población de estudio, en la realidad cada individuo presenta una curva de crecimiento propia, generando una fuente de variación asociada a cada $\beta$ i. El crecimiento animal está determinado por los efectos genéticos y/o ambientales, los cuales inciden de modo diferente a través del tiempo, con posibles repercusiones en la estructura de (co)varianzas, así como en la estimación de los $\beta$ i. La metodología de modelos no lineales mixtos (MNLM) se desarrolló con el propósito de mejorar el ajuste y la validación de los MNL, a partir de la estructura de (co)varianzas definida por los propios $\beta$ i como efectos aleatorios (Ching-Fan 2005, Zheng 2010).

En las regiones tropicales las poblaciones de bovinos son principalmente razas cebuínas o cruzas con éstas, debido a sus características de adaptación y producción bajo condiciones tropicales extensivas, donde la alimentación se basa en pastoreo (Burrow 2012). En Cuba, el ganado cebú representa la mayor proporción de bovinos productores de carne, el destete se realiza a los siete meses de edad, y cerca del $25 \%$ de los becerros se envía a las pruebas de comportamiento en pastoreo (Espinoza et al. 2007, Guillen et al. 2011). El análisis de los parámetros de crecimiento derivados de los MNL permite implementar y orientar programas específicos de alimentación, manejo y comercialización; así como definir esquemas de mejoramiento genético con el fin de modificar la curva de crecimiento. Por lo anterior el objetivo del presente estudio fue caracterizar el crecimiento del ganado cebú cubano con base en modelos no lineales vs modelos no lineales mixtos (MNLM), con posibles diferencias en el ajuste de los modelos y la estimación de parámetros de crecimiento.

\section{MATERIALES Y MÉTODOS}

En las pruebas de comportamiento $(\mathrm{PC})$ se toman cuatro mediciones del peso vivo, más el peso al destete, para generar una distribución de datos o pesadas a través del tiempo. La base de datos se conformó con 15990 registros de peso vivo, de 3 198 machos con cinco pesadas: 1) peso al destete, realizado de 150 a 229 d de edad; 2) peso al ini- 


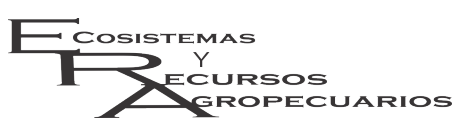

cio de la prueba de 163 a $286 \mathrm{~d}$; 3) primera pesada dentro de la prueba de comportamiento, medido entre los 283 y $427 \mathrm{~d}$; 4) segunda pesada dentro de la prueba, tomado entre 326 y $507 \mathrm{~d}$; y 5) peso al final de la prueba, realizado entre 398 y $630 \mathrm{~d}$ de edad. Se generó el peso ajustado al destete (PD, $210 \mathrm{~d})$, al inicio de la PC (PCl; $240 \mathrm{~d})$, así como el peso ajustado a los 365 (P365), 450 (P450) y $540 \mathrm{~d}$ (P540). En los procedimientos de ajustes, se utilizó la ganancia diaria de peso correspondiente a cada periodo; el PD se obtuvo a partir del peso al nacer (CONARGEN 2010).

La base de datos fue proporcionada por el Centro Nacional de Control Pecuario de la República de Cuba. Los datos provienen de cinco hatos donde se realizaron pruebas de comportamiento, a partir de alimentación con base en pastoreo de gramíneas tropicales (Cynodon nlemfuensis y Panicum máximum), en predios ubicados entre los 20 y $23^{\circ} \mathrm{LN}$, y 74 y $85^{\circ} \mathrm{LO}$, con dos estaciones claramente definidas; la de lluvias de mayo a octubre, en la que ocurre del 70 al $80 \%$ de la precipitación, y la estación seca de noviembre a abril. La temperatura media anual es de $23.1{ }^{\circ} \mathrm{C}$ y la humedad relativa de 60 a $70 \%$ durante el día y de 80 a $90 \%$ en la noche (Espinoza et al. 2007, IMRC 2015).

Para caracterizar el crecimiento se utilizaron cuatro MNL (Thornley y France 2007, Agudelo et al. 2008): Brody (BRO), Logístico (LOG), von Bertalanffy (BER) y Gompertz (GOM); los cuales están formados por tres coeficientes de regresión $(\beta 1, \beta 2, \beta 3)$. En las ecuaciones de los MNL, y representa el peso vivo medido al t tiempo; $\beta 1$, es el valor asintótico cuando $t$ tiende a infinito, interpretado como el peso adulto; $\beta 2$, es un parámetro de ajuste cuando y $\neq 0$ y $\mathrm{t} \neq 0 ; \beta 3$, es la tasa de crecimiento, expresando la ganancia de peso como proporción del peso total (Ratkowsky 1990, Blasco 1999). También se estimó el punto de inflexión (PI) en cada uno de los MNL (Tabla 1). Para contrastar los MNLM se realizaron los análisis ANA1 y ANA2 con el procedimiento NLMIXED y el método iterativo de Gauss (Wolfinger 1999) del programa SAS (SAS 2005). El análisis con MNLM (ANA1) incluyó
Domínguez-Viveros et al. Crecimiento de bovinos Cebú en Pastoreo Ecosist. Recur. Agropec. 4(11):341-348,2017

el efecto aleatorio correspondiente al $\beta 1$, más los residuales ( $\varepsilon \mathrm{ij}$ ); y el análisis con MNL (ANA2) no incluyó efectos aleatorios relativos a los coeficientes de regresión, solo los iij. Los efectos aleatorios de los zij y $\beta 1$, fueron de estructura no correlacionados y se ajustaron a las suposiciones de una distribución normal, con media igual a cero y varianzas $\sigma^{2} \varepsilon$ y $\sigma^{2} \beta 1$, respectivamente. No se incluyeron los efectos aleatorios relativos a $\beta 2$ y $\beta 3$, dado que en análisis previos se verifico que sus componentes de varianza fueron de baja magnitud y tienden a cero.

La selección del modelo con mejor ajuste se realizó en función de los criterios de : 1) error de predicción promedio $\left[E P P=\left(\sum_{i=1}^{n}\left(\frac{p v i-p e i}{p e i}\right) *\right.\right.$ $100) / n]$; 2) varianza del error de predicción $\left.\left[V E P=\sum_{i=1}^{n}(p e i-p v i)^{2} / n\right] ; 3\right)$ estadístico Durbin Watson $[D W=2(1-\rho) ; \rho=$ $\left.\frac{\sum_{t=2}^{n}\left(e_{t}-e_{t-1}\right)^{2}}{\sum_{t=1}^{n} e_{t^{2}}}\right]$; 4) coeficiente de determinación $[R 2=(1-(s c e / s c t))] ; 5)$ criterio de información Akaike $[A I C=-2 * \operatorname{Loglik}+2 k]$ y 6) criterio de información Bayesiano $[B I C=$ $-2 * \operatorname{Loglik}+\log (n) * k$. Donde: $p v i=$ peso vivo en la $i$ - ésima edad; $p e i=$ peso vivo estimado para la $i$ - ésima edad; $n=$ número total de datos; sce = suma de cuadrados de los residuales; $s c t=$ suma de cuadrados total; Log lik = logaritmo de la función de verosimilitud; $k=$ número de parámetros en el modelo (Motulsky y Christopoulos 2003, Torres et al. 2012, Palacios-Espinoza et al. 2016). El EPP analiza la relación que existe entre el peso medido y el peso estimado, en función del signo, el MNL sobreestima (+) o subestima (-) las predicciones. Para EPP, VEP, AIC y BIC, el modelo con el menor valor se consideró como de mejor ajuste; a diferencia del R2, el modelo con el valor más alto representa mejor ajuste. El DW analiza las auto correlaciones en los errores, con los planteamientos de si $2<\mathrm{DW}<4$ existe auto correlación negativa; si $0<\mathrm{DW}<2$ indica ausencia de auto correlación; y si $\mathrm{DW}<0$ indica que existe auto correlación positiva.

\section{RESULTADOS}

El peso promedio ( $\mathrm{kg} \pm$ desviación estándar) 
Tabla 1. Modelos no lineales utilizados para analizar el crecimiento de bovinos Cebú Cubano

\begin{tabular}{|c|c|c|}
\hline Modelo & Ecuacion & PI \\
\hline Brody & $y=\beta 1^{*}\left(1-\beta 2^{*}\left(\exp \left(-\beta 3^{*} t\right)\right)\right)$ & - \\
\hline Logístico & $y=\beta 1 /\left(1+\beta 2^{*}(\exp (-\beta 3 * t))\right)$ & $t=\ln \beta 2 / \beta 3$ \\
\hline von Bertalanffy & $y=\beta 1^{*}\left(\left(1-\beta 2^{*}\left(\exp \left(-\beta 3^{*} t\right)\right)\right)^{* *} 3\right)$ & $t=\ln 3 \beta 2 / \beta 3$ \\
\hline Gompertz & $y=\beta 1^{*}\left(\exp \left(-\beta 2 *\left(\exp \left(-\beta 3^{*} t\right)\right)\right)\right)$ & $t=\ln \beta 2 / \beta 3$ \\
\hline
\end{tabular}

Tabla 2. Resultados para los coeficientes de regresión ( $\beta$ i) y componentes de varianza a través de los modelos no lineales evaluados.

\begin{tabular}{lccccccc}
\hline Modelo & $\beta 1$ & \multicolumn{7}{c}{$\beta 2$} & $\beta 3$ & $\mathrm{PI}$ & $\sigma^{2} e$ & $\sigma^{2} \beta 1$ \\
\hline \multicolumn{7}{c}{ Análisis con modelos no lineales mixtos } \\
Brody & $388.4 \pm 3.2$ & $1.004 \pm 0.01$ & $0.00264 \pm 0.00005$ & - & 1871.4 & 1094.9 \\
Logístico & $392.9 \pm 3.0$ & $4.055 \pm 0.03$ & $0.00484 \pm 0.00005$ & 289 & 1220.1 & 1572.1 \\
von Bertalanffy & $388.7 \pm 3.3$ & $0.503 \pm 0.01$ & $0.00329 \pm 0.00006$ & 125 & 1792.6 & 1067.9 \\
Gompertz & $397.4 \pm 3.3$ & $1.898 \pm 0.01$ & $0.00353 \pm 0.00005$ & 181 & 1295.2 & 1654.8 \\
Análisis con modelos no lineales & & & \\
Body & $1659.3 \pm 0.00$ & $0.9619 \pm 0.01$ & $0.00030 \pm 0.00001$ & - & 2657.4 & - \\
Logístico & $1851.6 \pm 0.01$ & $16.89 \pm 0.11$ & $0.00226 \pm 0.00001$ & 1250 & 2558.3 & - \\
Gompertalanffy & $4939.7 \pm 0.01$ & $0.7359 \pm 0.01$ & $0.00036 \pm 0.00001$ & 2199 & 2581.6 & - \\
\hline$\sigma 2 e:$ varianza residual. $\sigma 2 \beta 1:$ varianza asociada al coeficiente de regresión $\beta 1 . \beta 1, \beta 2$ y $\beta 3:$ \\
coeficientes de regresión que conforman los modelos no lineales evaluados. PI = edad en días al \\
punto de inflexión de la curva de crecimiento.
\end{tabular}

Tabla 3. Resultados para los estadísticos utilizados en la selección de modelos.

\begin{tabular}{|c|c|c|c|c|c|c|}
\hline & EPP & VEP & $\mathrm{AIC}$ & $\mathrm{BIC}$ & $\mathrm{R} 2$ & DW \\
\hline & \multicolumn{6}{|c|}{ Análisis con modelos no lineales mixtos } \\
\hline Brody & -5.2 & 2851.9 & 167543 & 167573 & 94.96 & 1.14 \\
\hline Logístico & -4.3 & 2684.1 & 163443 & 163474 & 95.25 & 1.08 \\
\hline von Bertalanffy & -5.0 & 2789.8 & 166965 & 166995 & 95.07 & 1.10 \\
\hline \multirow[t]{2}{*}{ Gompertz } & -4.6 & 2748.8 & 164251 & 164292 & 95.14 & 1.10 \\
\hline & \multicolumn{6}{|c|}{ Análisis con modelos no lineales } \\
\hline Brody & -4.0 & 2649.8 & 171416 & 171447 & 95.31 & 1.07 \\
\hline Logístico & -3.9 & 2559.1 & 170865 & 170895 & 95.48 & 1.03 \\
\hline von Bertalanffy & -2.5 & 2594.1 & 170987 & 171018 & 95.41 & 1.04 \\
\hline Gompertz & -4.1 & 2568.2 & 170922 & 170953 & 95.46 & 1.04 \\
\hline
\end{tabular}

para PD, PCI, P365, P450 y P540 fue de $175.9 \pm$ $33.4,182.5 \pm 32.1,225.8 \pm 49.6,266.8 \pm 61.9$ y $340.3 \pm 68.1$, respectivamente. En el Tabla 2 se presentan los resultados para los coeficientes de regresión que conforman los modelos evaluados, así como los componentes de varianza de los efectos aleatorios en cada modelo. Mientras que en la Tabla 3 se describen los resultados para los estadísticos utilizados en la selección del modelo con mejor ajuste. Con base en los criterios de información AIC y BIC, los análisis con los MNLM (ANA1) presen- taron los mejores resultados, siendo el modelo LOG el que presentó mejor ajuste, seguido de los modelos de GOM y BER, y BRO. Para el R2 y el estadístico DW, los resultados fueron similares en los análisis, todos los modelos explicaron alrededor del $95 \%$ de la variación de los datos, lo que indica ausencia de auto correlación en los residuales. En todos los análisis el signo del EPP fue negativo, indicando una subestimación en la predicción del peso vivo; sin embargo, los análisis con base en los MNLM tuvieron mayores estimaciones del EPP y la VEP. 


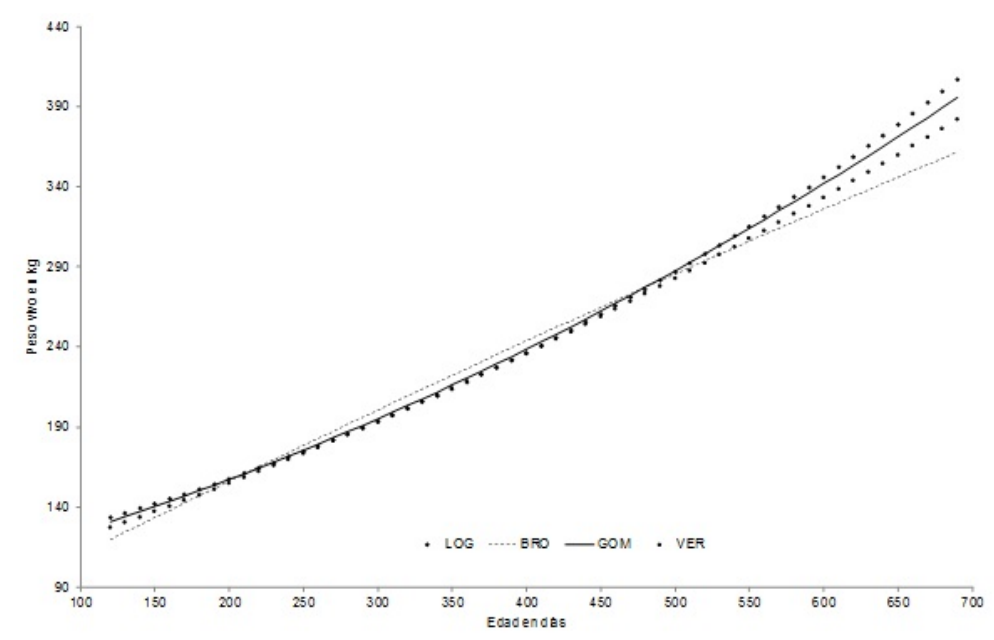

Figura 1. Curvas de crecimiento en bovinos Cebú Cubano a partir de los modelos no lineales: LOG: Logístico; BRO: Brody; GOM: Gompertz; y, BER: von Bertalanffy.

Las estimaciones de $\beta 1, \beta 3$ y $\mathrm{PI}$ como resultados del ANA2 (Tabla 2) están sobre estimadas y fuera de contexto para interpretarse 0 asociarse al crecimiento de los bovinos. En la Figura 1 se muestran las curvas de crecimiento elaboradas con los resultados del ANA2, donde se observa que los modelos generados no tienen capacidad predictiva con base en el proceso generador de los datos. Resultados diferentes se presentan en la Figura 2, a partir del ANA1 con base en MNLM, las curvas describen de forma adecuada el comportamiento del crecimiento a través del tiempo. El análisis de residuales a través de los estadísticos VEP, EPP y DW está asociado a la capacidad predictiva o proceso de validación del modelo; los criterios AIC, BIC y R2 corresponden a la jerarquización y nivel de ajuste de los modelos. Con base en los resultados de la Tabla 3, los MNLM del ANA1 presentaron mejor nivel de ajuste, buena capacidad de predicción, y los parámetros de crecimiento se apegan a una interpretación biológica.

\section{DISCUSIÓN}

En el análisis y la caracterización del crecimiento de bovinos Bos indicus, son diversos con respecto al MNL de mejor ajuste, capacidad predictiva e interpretación de los parámetros de crecimiento. Al respecto Paro et al. (2004) y de Andrade et al. (2010) coinciden con los resultados del presente estudio, ya qe el LOG fue el idóneo para describir y evaluar el crecimiento en las poblaciones evaluadas; mientras que de Lima et al. (2011) y da Silva et al. (2013) el BRO, y para Lopes et al. (2012) el BER, fueron los MNL de mejor ajuste. Estudios relacionados con la producción animal, donde los MNLM han presentado mejor ajuste con respecto a los MNL, fueron publicados por Palacios-Espinoza et al. (2016), Köhn et al. (2007) y Sofaer et al. (2013).

El PD, PCI, P365, P450 y P540 coinciden con la información de crecimiento en ganado Cebú analizado por Menéndez et al. (2006), EspinozaVillavicencio et al. (2008), Guillen et al. (2011), y Guillen et al. (2012). El crecimiento de los bovinos para carne está caracterizado por una curva de tipo sigmoidea de tres fases: un crecimiento lento inicial, una fase de aceleración pronunciada y un período de desaceleración, hasta alcanzar el peso adulto (Lawrence y Fowler 2002, Goyache 2005). En el contexto de curva de crecimiento tipo sigmoide, el punto de inflexión (PI), determina un cambio en la tasa de crecimiento y señala el momento en que el crecimiento deja de acelerar. La forma de la curva está determinada por la interacción del peso 


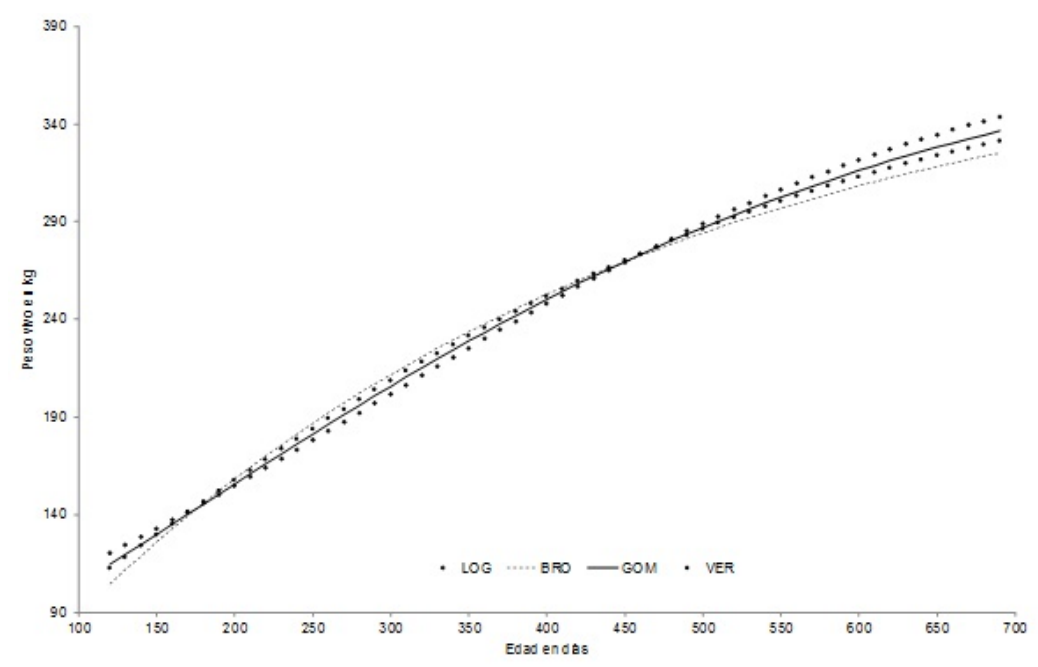

Figura 2. Curvas de crecimiento en bovinos Cebú Cubano a partir de los modelos no lineales mixtos: LOG: Logístico, BRO: Brody, GOM: Gompertz y BER: von Bertalanffy.

asintótico $(\beta 1)$ y la velocidad ( $\beta 3$ ) de crecimiento (Owens et al. 1993); que de manera conjunta con la inclinación de la curva, proporciona información sobre el comportamiento de los datos (Ratkowsky 1990, Balzarini et al. 2004).

El modelo LOG presenta una curva simétrica respecto al $\mathrm{Pl}$; el cual alcanza el $50 \%$ del peso asintótico $(\beta 1)$ y considera la velocidad de crecimiento proporcional (Goyache, 2005). Con base en los resultados de MNLM, para el modelo LOG, el PI (Tabla 1) se calculó alrededor de los $289 \mathrm{~d}$, el cual se ubicó en la primera pesada dentro de la prueba de comportamiento, a diferencia de los modelos BER y GOM, donde el PI está ubicado en el periodo predestete. En el modelo LOG los P365, P450 y P540 correspondieron al $57 \%, 67 \%$ y $86 \%$ del peso asintótico (392.9 kg); con base en la tasa de crecimiento, el peso asintótico se alcanza después de los 24 meses de edad. Se han explorado los posibles efectos sobre el ajuste y jerarquización de los MNL, dado la estructura de la base de datos, así como las características de las poblaciones de estudio (de Lima et al. 2011, Posada et al. 2011). De acuerdo con Arango y Van Vleck (2002) y Santoro et al. (2005), la dificultad de ajuste y capacidad de predicción de los MNL se debe al uso de ani- males jóvenes para la estimación del peso adulto, así como la falta de datos en periodos importantes de la curva de crecimiento; el punto para alcanzar el peso adulto no se define de forma correcta cuando la edad máxima de los datos analizados es de dos años, ya que aún falta desarrollo y conformación de los animales.

\section{CONCLUSIONES}

Todos los modelos explicaron alrededor del $95 \%$ de la variación de los datos, los valores del estadístico Durbin Watson indican que no hubo auto correlación en los residuales, y el signo en el error de predicción promedio indica que todos los modelos tienden a subestimar las predicciones. EI modelo logístico, derivado del análisis de modelos no lineales mixtos, fue el de mejor ajuste y capacidad de predicción. Los modelos no lineales mixtos presentaron el mejor ajuste, con base en los criterios de información Akaike y Bayesiano. Las curvas de crecimiento generadas con los modelos no lineales mixtos, presentaron buena capacidad predictiva. 


\section{LITERATURA CITADA}

Agudelo GA, Cerón MF, Restrepo BLF (2008) Modelación de funciones de crecimiento aplicadas a la producción animal. Revista Colombiana de Ciencias Pecuarias 20: 39-58.

Arango JA, Van Vleck LD (2002) Size of beef cows: early ideas, new developments. Genetics and Molecular Research 1: 51-63.

Balzarini M, Macchiavelli R, Casanoves F (2004) Aplicaciones de modelos mixtos en agricultura y forestería. Centro agronómico tropical de investigación y enseñanza - CATIE. Costa Rica. 189p.

Blasco A (1999) La descripción del crecimiento. Informe técnico ocasional No 6. Departamento de ciencia animal. Universidad Politécnica de Valencia. Valencia, España. 20p.

Burnham KP, Anderson DR (1998) Model selection and inference. Springer. London, UK. 38p.

Burrow HM (2012) Importance of adaptation and genotype $\times$ environment interactions in tropical beef breeding systems. Animal 6: 729-740.

Ching-Fan S (2005) Using SAS PROC NLMIXED to fit item response theory models. Behavior Research Methods 37: 202-218.

CONARGEN (2010) Guía técnica de programas de control de producción y mejoramiento genético en bovinos de carne. Consejo Nacional de los Recursos Genéticos Pecuarios. México, DF. 80p.

da Silva MKN, Ribeiro de FA, da Silva FAJ, Ferreira DFE (2013) Nonlinear models for fitting growth curves of Nellore cows reared in the Amazon Biome. Revista Brasileira de Zootecnia 42: 645-650.

de Andrade SL, Novais CD, Souza CPL, Mendes MCH, Martins FR (2010) Curvas de crescimiento em bovinos de raça Indubrasil criados no Estado do Sergipe. Revista de Ciencias Agronómica 41: 671-676.

de Lima SF, Mello de AM, Ribeiro de FA, Umberto PI, Barreto MG (2011) Curvas de crescimiento em vacas de corte de diferentes tipos biológicos. Pesquisa Agropecuaria Brasileira 46: 262-271.

Espinoza VJL, Palacios EA, de Luna PR, Ávila SN, Guerra ID, González-Peña D, et al. (2007) Componentes de (co)varianza para caracteres de crecimiento y reproducción en ganado cebú en Cuba. Archivos de Zootecnia 56: 919-927.

Espinoza-Villavicencio JL, Palacios-Espinoza A, Guerra-Iglesias D, González-Peña D, Ortega-Pérez R, Rodrí guez-Almeida F (2008) Comparación de dos modelos para la estimación de parámetros y valores genéticos del peso en ganado cebú. Agrociencia 42: 29-36.

Guillén TA, Guerra ID, Ávila SN, González-Peña D, Palacios EA, de Luna PR, et al. (2011) Parámetros genéticos de rasgos de crecimiento en el ganado Cebú cubano. Revista Cubana de Ciencia Agrícola 45: 117-120.

Guillén TA, Guerra ID, Ávila SN, Palacios EA, Ortega PR, Espinoza VJL (2012) Parámetros y tendencias genéticas del peso al destete y a los 18 meses de edad en ganado Cebú bermejo de Cuba. Revista Mexicana Ciencias Pecuarias 3: 19-31.

Goyache FM (2005) Crecimiento, consumo y medidas corporales. Estandarización de las metodologías para evaluar la calidad del producto (animal vivo, canal carne y grasa) en los rumiantes. Monografías INIA - Serie ganadera. Madrid, España. 14p.

IMRC (2015) Instituto de meteorología de la República de Cuba. Disponible: http://www.met.inf.cu/. Fecha de consulta 15 noviembre de 2015. 
Köhn F, Sharifi AR, Simianer H (2007) Modeling the growth of the Goettingen minipig. Journal Animal Science 85: 84-92.

Lawrence T, Fowler V (2002) Growth of farm animal. 2a ed. CAB International. London UK. 478p.

Lopes FB, da Silva MC, Marques GE, McManus CM (2012) Analysis of longitudinal data of beef cattle raised on pasture from northern Brazil using nonlinear models. Tropical Animal Health Production 44: 1945-1951.

Menéndez BA, Guerra D, Planas T, Ramos F (2006) Factores que afectan el crecimiento de machos jóvenes de la raza Cebú en pruebas de comportamiento en condiciones de pastoreo. Revista Cubana de Ciencia Agrícola 40: 389-396.

Motulsky H, Christopoulos A (2003) Fitting models to biological data using linear and nonlinear regression. A practical guide to curve fitting. Graph Pad Software Inc. USA 351p.

Owens FN, Dubeski P, Hanson CF (1993) Factors that alter the growth and development of rumiants. Journal Animal Science 71: 3138-3150.

Palacios-Espinoza A, Domínguez-Viveros J, Padrón-Quintero Y, Rodríguez-Castro M, Rodríguez-Almeida FA, Espinoza-Villavicencio JL, et al. (2016) Caracterización de la curva de lactancia de bovinos siboney con modelos no lineales mixtos. Revista Mexicana de Ciencias Pecuarias 7: 233-243.

Paro de PCC, Umberto PI, Ribeiro de FA, Tambasco-Talhari D, de Almeida RLC, Mello de AM, et al. (2004) Ajuste de modelos não-lineares em estudios de associação entre polimorfismos genéticos e crescimiento em bovinos de corte. Revista Brasileira de Zootecnia 33: 1416-1425.

Posada OS, Rosero NR, Rodríguez N, Acosta A (2011) Estimación de parámetros de curvas de crecimiento de ganado Nellore criado en confinamiento. Revista MVZ Córdoba 16: 2701-2710.

Ratkowsky DA (1990) Handbook of nonlinear regression models. Marcel Dekker. New York, EUA. 350p.

Santoro KG, Paes BSB, de Albuquerque BLH, de Souza SE (2005) Estimativas de parâmetros de curvas de crecimiento de bovinos zebu, criados no Estado de Pernambuco. Revista Brasileira de Zootecnia 34: 2262-2279.

SAS (2005) Statistic Analysis System. User's Guide: Statistics (version 9.0). SAS Institute Inc. Cary NC, USA.

Sofaer HR, Phillip L, Chapman T, Scott S, Cameron K (2013) Advantages of nonlinear mixed models for fitting avian growth curves. Journal of Avian Biology 44: 469-478.

Thornley J, France J (2007) Mathematical models in agriculture, quantitative methods for plant, animal and ecological sciences. 2a Ed. CAB International, London UK. 845p.

Torres V, Barbosa I, Meyer R, Noda A, Sarduy L (2012) Criterios de bondad de ajuste en la selección de modelos no lineales en la descripción de comportamientos biológicos. Revista Cubana de Ciencia Agrícola 46: 345-350

Wolfinger RD (1999) Fitting nonlinear mixed models with the new NLMIXED procedure. SAS Users Group International Conference 24: 278-284.

Zheng M (2010) Fitting linear and nonlinear growth curve models using PROC NLMIXED. SAS Statistic and data analysis. Los Ángeles, EUA. 125 p.

Zucchini W (2000) An introduction to model selection. Journal Mathematic Psychology 44: 41-61. 